\title{
More on the Robust Solution for Epidemiology: Nineteenth-Century Quebec
}

\section{Paul TE Cusacki*}

Brealey Drive, Peterborough, 77432, Infrobright, Canada

\begin{abstract}
Here we consider the Robust Solution as applied to the cholera epidemic in Lower Canada (Quebec) in 1832. We find that the mathematics from that procedure provides the mathematical foundation or the study. The rate of growth of the virus must be kept below $14 \%$ to terminate the spread of the disease.
\end{abstract}

Keywords: Energy; Time; Density; The bell normal; The golden mean parabola

\section{Introduction}

This paper is an examination of the mathematics already wellestablished as the Robust solution. We use this solution applied to the cholera epidemic, particularly in what is toddy, Quebec-Montreal and Quebec City. The data was found in Bilson's book, A Darkened house, Cholera in nineteenth Century Canada. Some figures come from the Saint John Cholera epidemic 1854 [1-3]. We begin there.

In Saint John, 1854,

1103 deaths from Cholera/pop. 30,000 $=1 / \mathrm{e}=\mathrm{e}^{\wedge}-\mathrm{t}=\mathrm{E}$

In Quebec 1832-33:

$3451 / \mathrm{X}=1 / \mathrm{e}$

$\mathrm{X}=1269=$ rho $=$ density $\sim 4 / \mathrm{Pi}$

$1269=78.8$ deaths $/ 1000$.

In Montreal, there was a cholera death rate of $74 / 1000$. In Quebec City, Twas a cholera death rate of 82/1000. Average $(74+82) / 1000=41 / 1000[4,5]$.

Now $\mathrm{rho} / \mathrm{c}=126.9 / 2.9979=0.4235 \sim \mathrm{Pi}-\mathrm{e}=0 . / 4233 .=$ Resistance to Disease $=\mathrm{Rd}$

[Deaths/1000]=rho

$\mathrm{Rho} / \mathrm{c}=\mathrm{cuz}$

$\mathrm{Rho} / \mathrm{c}^{\star} \mathrm{Pi}=$ Space $\mathrm{s}$

And, from Astro-theology mathematics:

The cross-product vector is:

$\mathrm{S}=|\mathrm{E}| \mathrm{t} \mid \cos$ theta

Resistance to death $=(\mathrm{Vd})($ Cycle $) \cos ($ Cycle $)$

$=\mathrm{e}^{\star}(40 \%$ of a cycle $) \cos (1 \mathrm{rad})$

$=58.75 \%$

$58.75 \%=\mathrm{Pi}=54.18 \%$

$1-54.18 \%=45.82 \% \sim 45.7=$ death rate in the entire province of Quebec

\section{$1-58.75 \%=41.25 \%$}

Cf Average Death rate above $=41 / 1000$.
$\mathrm{Re}=\mathrm{rho} \mathrm{v} / \mathrm{Nu}$

(127(0.8415)/0.27=395 solving:

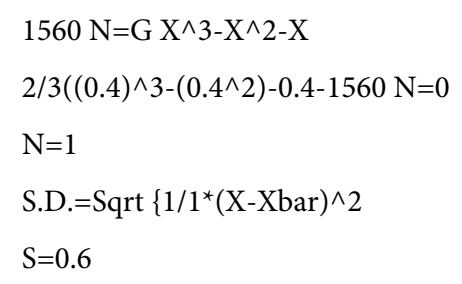

Mew bar $=1.2$

$1.2 * c^{\wedge} 2=1.08=Z$ score for $85.99 \% 1 / 85.99=116.29=$ Mass no of elements in the periodic table.

$1-0.8599=0.14=14 \%$ minimum profit to sustain growth .

Finally,

$\mathrm{E}=\mathrm{Mc}^{\wedge} 2$

*Corresponding author: Cusack P, Brealey Drive, Peterborough, 77432, Infrobright, Canada, Tel: (506) 214-331; E-mail: St-michael@hotmail.com

Received March 02, 2017; Accepted April 22, 2017; Published April 25, 2017

Citation: Cusacki PTE (2017) More on the Robust Solution for Epidemiology: Nineteenth-Century Quebec. J Biom Biostat 8: 342. doi:10.4172/21556180.1000342

Copyright: @ 2017 Cusacki PTE. This is an open-access article distributed under the terms of the Creative Commons Attribution License, which permits unrestricted use, distribution, and reproduction in any medium, provided the original author and source are credited. 


$$
\begin{aligned}
& =(1 / \mathrm{e})\left((2.997929)^{2}\right. \\
& =3.3063 \\
& \mathrm{E}=1 / \mathrm{t} \\
& \mathrm{t}=1 / \mathrm{E}=1 / 3.3=0.302
\end{aligned}
$$

Root for the Bell Normal.

$\Phi=1 / \sqrt{ }(\sigma 2 \pi) \mathrm{e}^{-1 / 2[(\mathrm{X}-1.30 / 1.30)]^{2}}$

Root $\mathrm{X}=\mathrm{t}=3.02$

$1 / \mathrm{c}=\mathrm{Mc}^{2}$

$1=\mathrm{Mc}^{3}$

$=99.125=1 / 1.009 \sim 1.01=\mathrm{E}$

$\mathrm{E}=\Phi$

Roots $\mathrm{X}=0,1.4$

$0 \leq \mathrm{X} \leq 1.4$

$1-0.8599=0.1401$

\section{$\mathrm{Z}=1.08$}

$1 / 81.99=116.29=$ Mass of final element in periodic table.

$\mathrm{Y}=\mathrm{e}^{-\mathrm{t} \cos \mathrm{t}}$

$116.29=\mathrm{e}^{\mathrm{t}} \cos \mathrm{t}$

$\mathrm{Y}=1 / \mathrm{e}=\mathrm{E}$

Refer to Figure 1.

$\mathrm{t}^{2}={ }^{2}-1=0$

$\mathrm{dE} / \mathrm{dt}=2 \mathrm{t}-1=1$

$2=1$

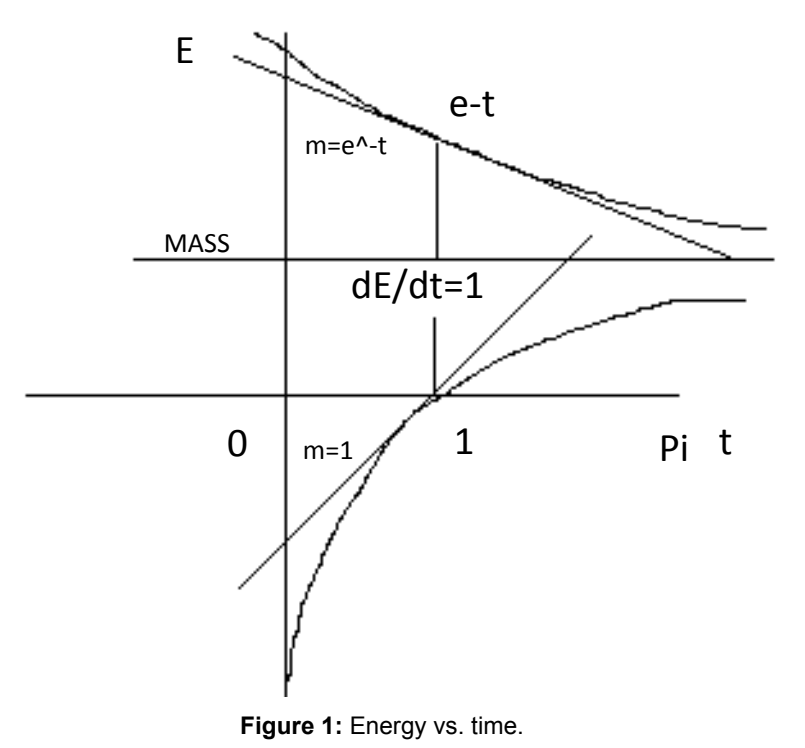

$$
\begin{aligned}
& \mathrm{E}=1 / \mathrm{e}^{-\mathrm{t}} \\
& \mathrm{E}=1 / \mathrm{e}^{-1}=2.718=\mathrm{Y} \\
& \text { At } \mathrm{t}=0, \text { Ln } \mathrm{t}=0 \\
& \mathrm{t}=1 \\
& \text { At } \mathrm{t}=\pi \\
& \text { Ln } \pi+\mathrm{cuz}=2.568 \sim \pi / 2=\mathrm{t} / 2 \\
& \mathrm{t} / 2=1 / 2=\mathrm{Emin}=-1.25 \\
& \text { And } \\
& \text { Ln } \pi+0.4233=1 / \mathrm{e}^{\mathrm{t}} \\
& \mathrm{G} \sim 6.54=1 / \mathrm{e}^{\mathrm{t}} \\
& \mathrm{e}^{\mathrm{t}}=0.1529=1-\sin 1=\text { Moment } \\
& 1-0.1529=0.8471 \\
& \sin ^{-1}(0.8471)=57.89^{\circ}=1.01 \mathrm{rads}=\mathrm{E} .
\end{aligned}
$$

\section{Conclusion}

Like every other two pole problem (infected or not infected) the two-pole solution works to solve problems in epidemiology. Keeping the growth rate below $14 \%$ will terminate a pandemic.

\section{References}

1. Somerville GF (1854) Saint John and Portland cholera Deaths. Saint John, NB.

2. Bilson G (1980) A Darkened House, Cholera in 19th Century Canada University of Toronto Press.

3. Bislon G (1854) The Cholera Epidemic in Saint John, NB, Acadiensis.

4. Cusack P (2015) Cholera and Mental Illness, Mental health, Family Medicine.

5. Cusack P (2016) Astrotheology Cusack's Universe. J of Physical Mathematics OMI Jan 2016. 